\title{
Determination of Camera Modulation-transfer Function by Electron Holography
}

\author{
Robert A. McLeod ${ }^{\dagger * \ddagger}$ and Marek Malac ${ }^{\dagger *}$ \\ ${ }^{\dagger}$ Department of Physics, University of Alberta, Edmonton, Alberta, Canada T6G 2G7. \\ *National Institute for Nanotech., 11421 Saskatchewan Dr., Edmonton, Alberta, Canada T6G 2M9. \\ †Corresponding author: rmcleod@phys.ualberta.ca
}

The point-spread function (PSF) and its frequency-space representation, the modulation-transfer function (MTF), of CCD cameras are a measure of CCD contribution to information spread and loss of contrast in imaging, EELS spectra, and electron holography in the TEM. It has previously been reported for TEM that the PSF has a narrow full-width half-maximum (FWHM), while the fullwidth tenth-maximum (FWTM) is quite broad which reduces the observed contrast by approximately a factor of three [1].

The two most popular existing methods for determination of MTF in the TEM are the deterministic knife-edge method and stochastic white noise method. However, the edge and noise methods are not equivalent and there is no direct correspondence between the two results. With the edge method, there is uncertainty associated with both the steepness of the edge (typically a beam stop) and its transverse smoothness [2]. In addition, the edge method usually only surveys a small fraction of the camera area at a fixed orientation relative to the CCD pixel axes. The noise method is known to overestimate the MTF compared to the edge method [3].

We present a deterministic method for measuring the modulation transfer function (MTF) directly from the contrast of off-axis holography interference fringes. This method creates a regular cosine fringe field of controllable frequency and orientation that is directly analogous to lattice fringes seen in high-resolution TEM. This method was previously proposed to support noise MTF measurement but was not considered a complete method as it could not measure the MTF down to frequencies approaching zero [4]. However, in extending to low spatial frequencies, we have found that the measured fringe contrast does begin to stabilize around 72 pixels per fringe (which corresponds to a magnification of $x 2.54 \mathrm{M}$ ), such that the maximum visibility can be extrapolated by a least squares fit.

Reference hologram series (i.e. no specimen) were taken on a Hitachi HF3300 instrument equipped with a cold-field emission gun, the double bi-prism configuration [5], and Gatan Ultrascan 1000 camera at 300 and $100 \mathrm{kV}$. The use of the double bi-prism configuration suppresses the Fresnel fringes normally apparent in single bi-prism holography and provides a more accurate estimation of both pixel period and fringe contrast. The pixel frequency was varied from 0.28 to 0.014 pix $^{-1}$ by adjusting the excitation of the projector lenses while bi-prism bias was held constant. At each pixel frequency, a series of ten holograms (Fig. 1) was taken to establish error in measured visibility. Visibility was determined by separately reconstructing the side-band and centre-band signals and finding the ratio of the two (Fig. 2) [6]. Histograms of the resulting visibility maps were taken to separate the fringeless pixels from the interference region (Fig. 3) and determine the mean visibility for a given hologram. The results across the pixel frequency range provide data for the MTF fit (Fig. 4). In agreement with Zuo (2000) [7], we found that MTF improves with decreasing accelerating voltage. 


\section{References}

[1] Thust, A. Phys Rev Lett. 102 (22) (2009): 220801-4.

[2] Mooney, P. E. Microsc Microanal. 15 (suppl. S02) (2009): 234-235.

[3] Meyer, R. R. and A. I. Kirkland. Microsc Res Tech. 49 (3) (2000): 269-280.

[4] de Ruijter, W. J. and J. K. Weiss. Rev Sci Instrum. 63 (10) (1992): 4314-4321.

[5] Harada, K. et al. Appl Phys Lett. 84(17) (2004): 3229-3232.

[6] Lehmann, M. and H. Lichte. Microsc Microanal. 8 (suppl. S02) (2002): 34-35.

[7] Zuo, J. M. Microsc Res Tech. 49 (3) (2000): 245-268.

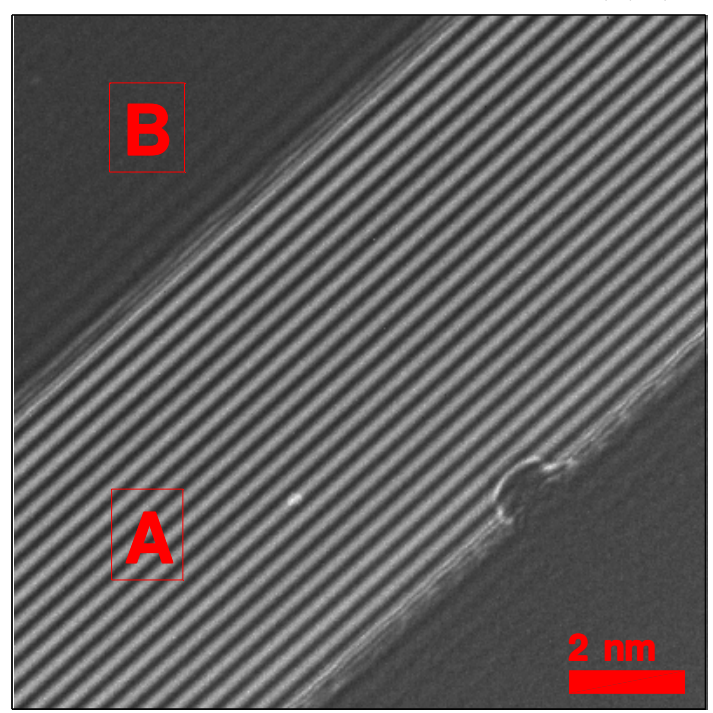

Figure 1: Sample hologram at pixel frequency $0.018 \mathrm{pix}^{-1}$, with a corresponding fringe period of $0.4 \mathrm{~nm}$ at the specimen plane. Section A indicates interference region and $\mathrm{B}$ the dark region.

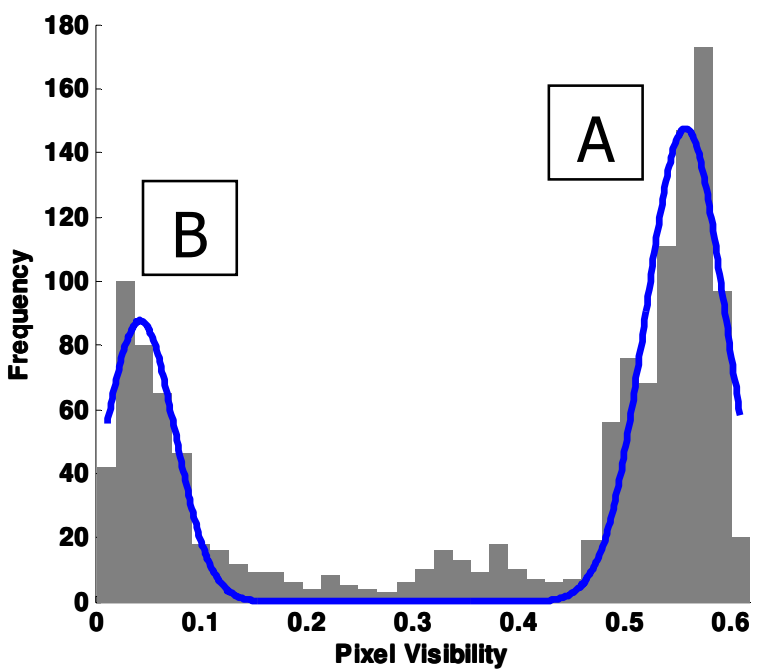

Figure 3: Histogram of visibility map in Fig. 2 shows bimodal distribution of fringe and fringeless pixels. The histogram was fitted with the sum of two Gaussian curves, with the high visibility peak having $\mathrm{V}=0.557 \pm 0.05$.

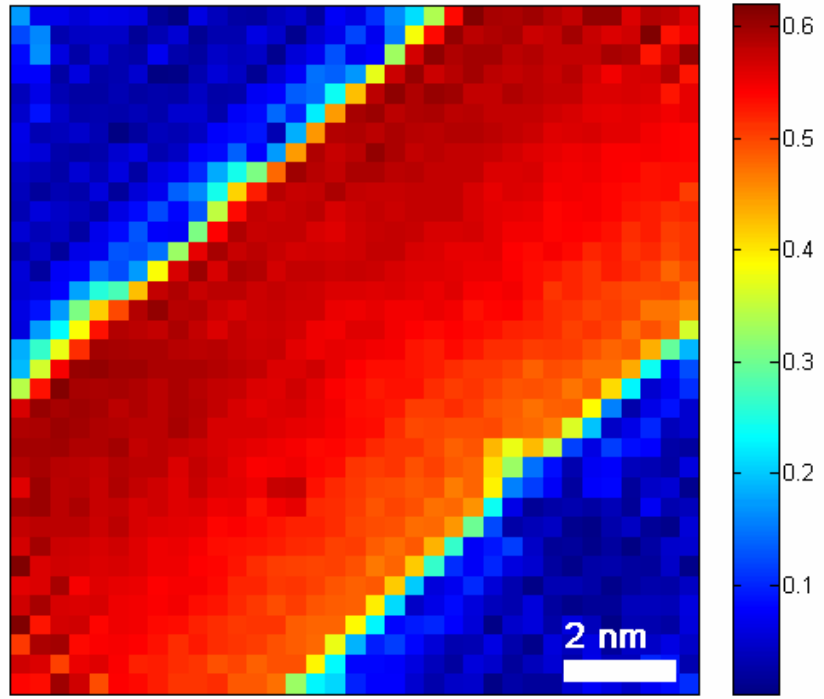

Figure 2: Map of fringe visibility from the hologram in Fig. 1, with the fringing field clearly evident and near zero visibility outside.

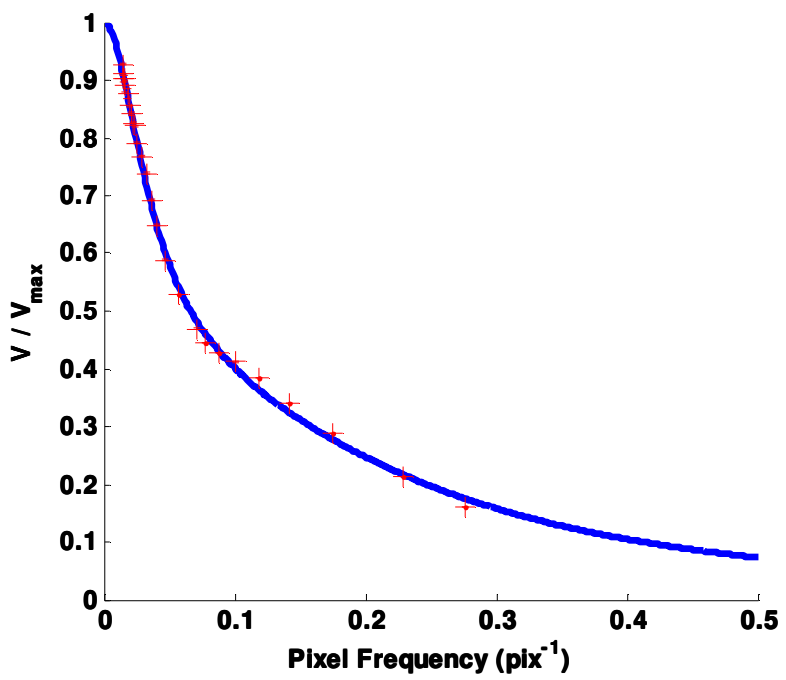

Figure 4: Mean experiment MTF (crosses) with fit to sum of two Lorentzian curves (solid line, $\mathrm{R}^{2}=0.998$ ). $\mathrm{V}_{\text {max }}=0.64$ for the applied bi-prism biases. The corresponding PSF has a FWHM of 3.7 pix and FWTM of 19.8 pix. 